\title{
Reduction in Flux Loss of a Nd-Fe-B Bonded Ring Magnet for a SPM Motor
}

\author{
T. Yanai ${ }^{1}$, S. Horita ${ }^{1}$, M. Nakano ${ }^{1}$, S. Tsutsumi ${ }^{2}$ and H. Fukunaga ${ }^{1}$, Member, IEEE \\ ${ }^{1}$ Guraduate School of Engineering, Nagasaki University, Nagasaki 852-8521, Japan \\ ${ }^{2}$ Appliacnces Company, Panasonic Corporation, Daito, Osaka 574-0044, Japan
}

\begin{abstract}
We have previously proposed a simulation method of an initial flux loss in permanent magnets using finite element method, and confirmed that predicted flux loss of a Nd-Fe-B boned ring magnet showed good agreement with measured flux loss. In this study, we applied our proposed method to a Nd-Fe-B boned ring magnet in a SPM motor. We modeled a rotor composed of a Nd-Fe-B bonded ring magnet and a soft magnetic core made from silicon steels, and carried out the simulations for magnetizing process and prediction process of the flux loss. From the simulation results, we found that the inner side of the ring magnet has large flux loss. In order to reduce in the flux loss, we enhanced the coercivity in the inner side of the ring magnet by partial replacement with another bonded magnet with high coercivity. Consequently, we found that the ring magnet with locally enhanced coercivity is effective to reduce in the flux loss.
\end{abstract}

Index Terms - Flux loss, Nd-Fe-B, Bonded magnet, SPM motor

\section{INTRODUCTION}

A lthough a Nd-Fe-B magnet has excellent magnetic properties at room temperature, it is pointed out that (1) the deterioration of the magnetic properties under a high operating temperature and (2) the flux loss after high temperature exposure are larger than those for other types of permanent magnets [1], [2]. Therefore, it is necessary to consider deterioration of the magnetic properties and large flux loss when Nd-Fe-B magnets apply to electric devices which operate under a high temperature. Since a prediction of the flux loss is one of useful techniques to design the shape of the magnet in the devices, we have measured the flux losses of various bonded magnets and studied a prediction method of the flux loss using a finite element method [3]-[7]. In our previous studies, we confirmed the good agreement between predicted flux loss and measured one in the Nd-Fe-B boned ring magnet, and clarified the importance of consideration of magnetizing process for accurate prediction of the flux loss. In this study, we applied our proposed method to a Nd-Fe-B bonded ring magnet for a SPM motor, and investigated the reduction in the flux loss of the ring magnet.

\section{ANALYSIS PROCEDURE}

We analyzed the flux loss in a Nd-Fe-B bonded ring magnet for a SPM motor using a Finite Element Method (FEM). Our previously-proposed method for the prediction of the flux loss is composed of following four steps,

(1) Simulation of magnetizing process

(2) Determination of demagnetization curves at room and exposure temperature

Manuscript received March 7, 2014. Corresponding author: S. Horita (email: bb52114244@cc.nagasaki-u.ac.jp).

Digital Object Identifier inserted by IEEE
(3) Simulation of distributions of magnetization at room and exposure temperature

(4) Prediction of distribution of flux loss.

We used two bonded magnets made from different magnetic powders of MQP-15-7 and MQP-B for the simulation. In this paper, the bonded magnets are described as Magnet \#1 (MQP-15-7) and Magnet \#2 (MQP-B), respectively. Figure 1 shows hysteresis loops of Magnet \#1 and Magnet \#2. Magnet \#2 has larger coercivity value compared with that for Magnet \#1, and used for an investigation of the effect of local enhancement in the coercivity in the ring magnet made from Magnet \#1. The magnetic properties of Magnet \#1 and Magnet \#2 are summarized in Table I.

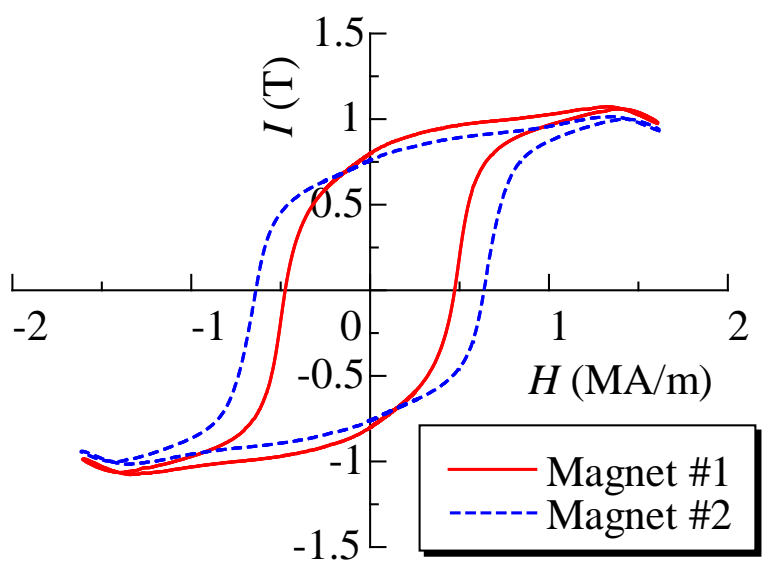

Fig.1 Hysteresis loops of Nd-Fe-B bonded magnets.

TABLE I. MAGNETIC PROPERTIES OF THE BONDED MAGNETS.

\begin{tabular}{ccc}
\hline \hline & Magnet \#1 & Magnet \#2 \\
\hline$I_{r}(\mathrm{~T})$ & 0.80 & 0.76 \\
$H_{c j}(\mathrm{kA} / \mathrm{m})$ & 470 & 635 \\
$H_{c b}(\mathrm{kA} / \mathrm{m})$ & 353 & 426 \\
$(\mathrm{BH})_{\max }$ & 78 & 84 \\
\hline \hline
\end{tabular}




\section{A. Simulation of magnetizing process}

Figure 2 shows a simulation model for the first step (magnetization analysis). A rotor consists of a Nd-Fe-B bonded ring magnet and a soft magnetic core made from silicon steels, and the ring magnet is arranged on the surface of the core. The inner and outer radii of the ring magnet are $23.4 \mathrm{~mm}$ and $25.15 \mathrm{~mm}$, respectively. We placed the rotor in a magnetizer which consists of a silicon steel yoke and exciting coils, and applied a magnetization current of $44 \mathrm{kA} \cdot$ turn to the coils. Consequently, we obtained the magnetized ring magnet with 8 magnetic poles.

\section{B. Determination of demagnetization curves at room and exposure temperature}

After the magnetizing process, we determined the coercivity and the demagnetization curves of each element in the magnet. The coercivity of each element was determined individually based on the measured relationship between the coercivity and the maximum applied field (Fig. 3). The demagnetization curves were also determined individually based on the relationship between the demagnetization curves and the experienced maximum applied field (Fig. 4). For Magnet \#2, both relationships were also obtained by the same measurements for Magnet \#1.

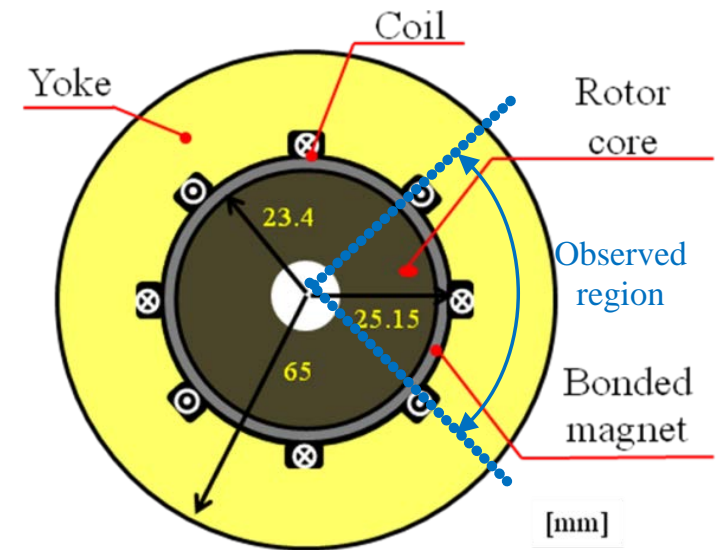

Fig.2 Simulation model used in the magnetization analysis. A rotor for a SPM motor and a magnetizer were modeled. The rotor consists of a Nd-Fe-B bonded ring magnet and a soft magnetic core made from silicon steels.

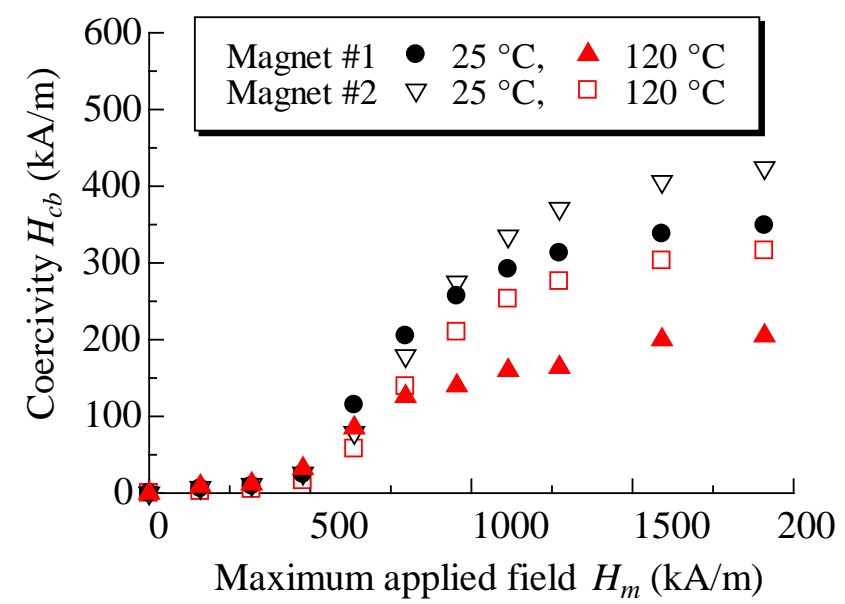

Fig.3 Relationships between the coercivity and the maximum applied field.

\section{Simulation of distributions of magnetization at room and exposure temperature}

From the simulations of the steps 1 and 2, we obtained the values of the coercivity and the demagnetization curves of each element in the magnet. In this step, we defined the coercivity and the demagnetization curve to each element as material properties, and then carried out the FEM analysis of magnetic field. As a result, we obtained the distributions of the magnetization in the magnet.

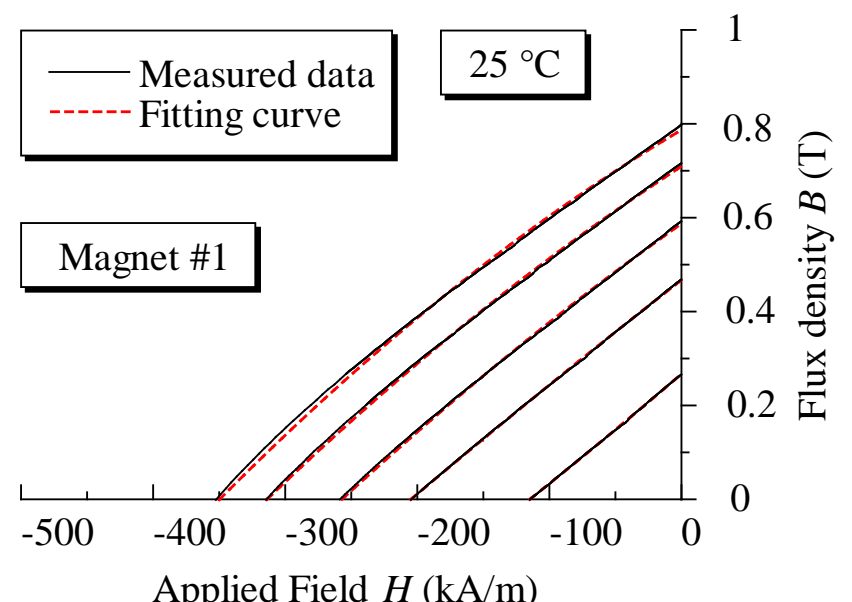

(a) Room temperature $\left(25^{\circ} \mathrm{C}\right)$

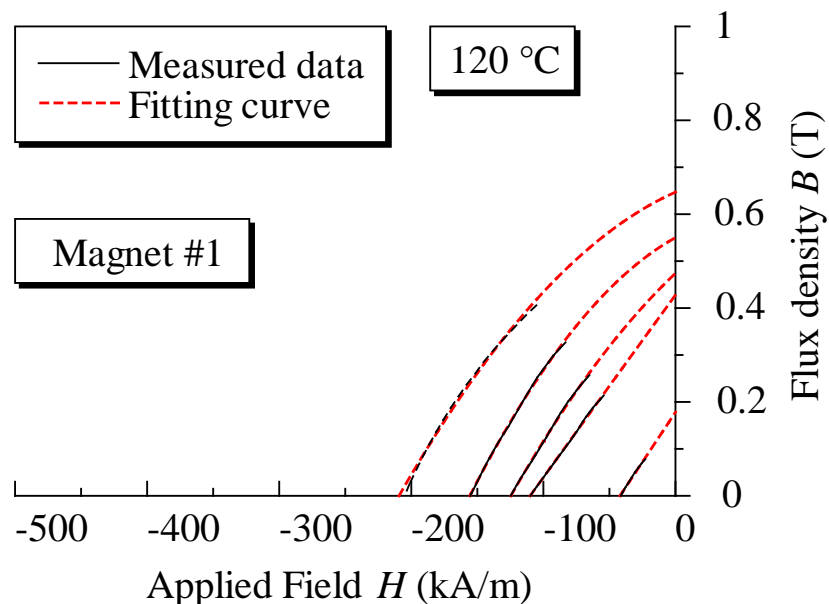

(b) High temperature $\left(120^{\circ} \mathrm{C}\right)$

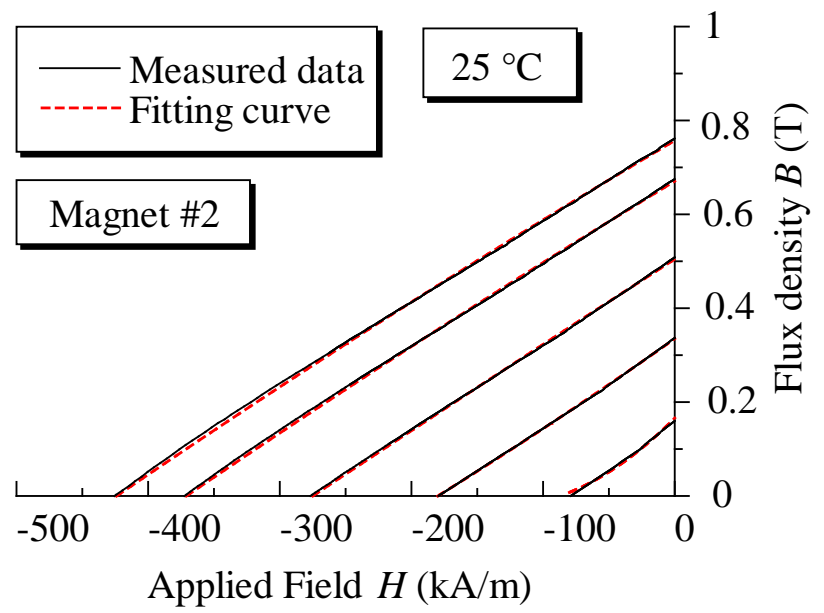

(c) Room temperature $\left(25^{\circ} \mathrm{C}\right)$ 


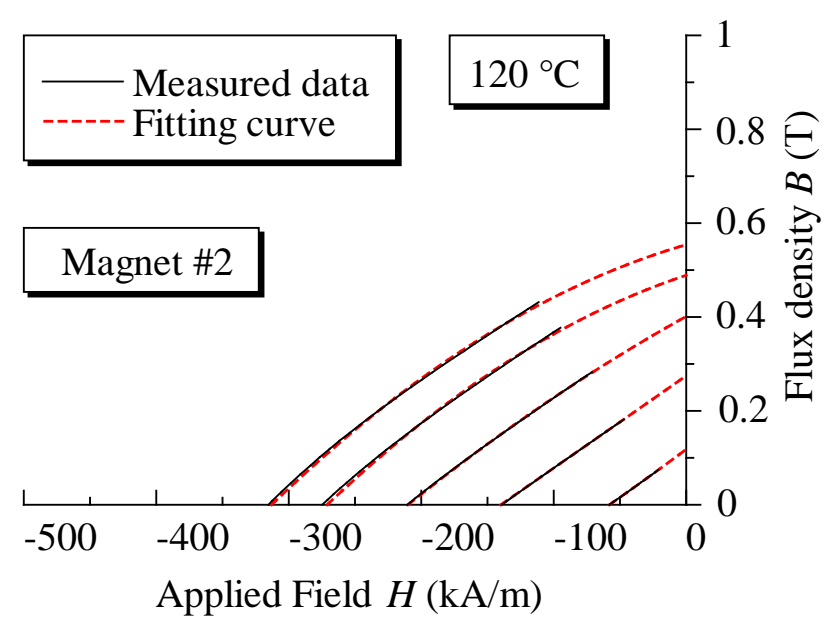

(d) High temperature $\left(120^{\circ} \mathrm{C}\right)$

Fig. 4 Measured demagnetization curves of Magnet \#1 and Magnet \#2 at 25 ${ }^{\circ} \mathrm{C}$ and $120{ }^{\circ} \mathrm{C}$. The fitting curves are also shown in this figure. The experienced maximum applied fields were 637, 796, 955, 1273, $1910 \mathrm{kA} / \mathrm{m}$

\section{Prediction of distribution of flux loss}

The flux loss $F L$ in the magnet is calculated by the following equation [3].

$$
F L=\frac{1-I_{w}\left(T_{e x}\right)}{I_{w}\left(T_{R T}\right)\left\{1-\alpha\left(T_{e x}-T_{R T}\right)\right\}}
$$

Where $I_{w}\left(T_{R T}\right)$ and $I_{w}\left(T_{e x}\right)$ are the magnetizations at room temperature $T_{R T}$ and the exposure temperature $T_{e x}$, respectively. The values of $I_{w}\left(T_{R T}\right)$ and $I_{w}\left(T_{e x}\right)$ obtained in the step 3 were used for the calculation of the flux loss. $\alpha$ is a temperature coefficient of the magnetization, and the measured $\alpha$ values of Magnet \#1 and Magnet \#2 are $9.75 \times 10^{-4} \mathrm{~K}^{-1}$ and $9.36 \times 10^{-4}$ $\mathrm{K}^{-1}$, respectively.

\section{RESULTS AND DISCUSSION}

\section{A. Flux loss in the ring magnet made from Magnet \#1}

Figure 5 shows the distribution of the flux loss and the demagnetizing field in the ring magnet made from Magnet \#1. Since the simulation model has periodicity, we only showed the distributions for "Observed region" shown in Fig.2. As shown in Fig.5, large flux loss was observed in the inner side of the ring magnet, and the regions with large flux loss were correlated with the regions with large demagnetization filed. This correlation agrees with the result for our previous study [4], [7], and suggests that we should pay attention to the regions with large demagnetization field to reduce in the flux loss.

\section{B. Flux loss in the ring magnet with locally enhanced coercivity}

It is well-known that a magnet with high coercivity has high thermal stability [8], [9]. Therefore, we considered that an enhancement of the coercivity in the regions with large demagnetization field is effective to reduce in the flux loss. In order to clarify the effect of the enhancement in the coercivity on the reduction in the flux loss, we enhanced the coercivity in the ring magnet by partial replacement of inner side of Magnet \#1 with Magnet \#2, and evaluated the flux loss.

The thicknesses of Magnet \#1 and Magnet \#2 are $1.25 \mathrm{~mm}$ and $0.5 \mathrm{~mm}$, respectively. The volume fraction of Magnet \#2 in the ring magnet is approximately 8 vol.\%. Figure 6 shows flux densities on the rotor surface for the ring magnets without or with locally enhanced coercivity. The angle from -45 to 45 degree corresponds to the angle of "Observed region" shown in Fig. 2. Both ring magnets showed almost the same waveforms of the flux density, and we confirmed that the flux density is not affected by the enhancement in the coercivity.

Figure 7 shows the distribution of the flux loss in the ring magnet (a) before and (b) after the enhancement in the coercivity. We confirmed that the enhancement in coercivity enables us to reduce in the flux loss in the ring magnet. The average value of the flux loss in the ring magnet with locally enhanced coercivity was $29.9 \%$, and this value was smaller than $37.5 \%$ for the ring magnet without the enhanced coercivity.

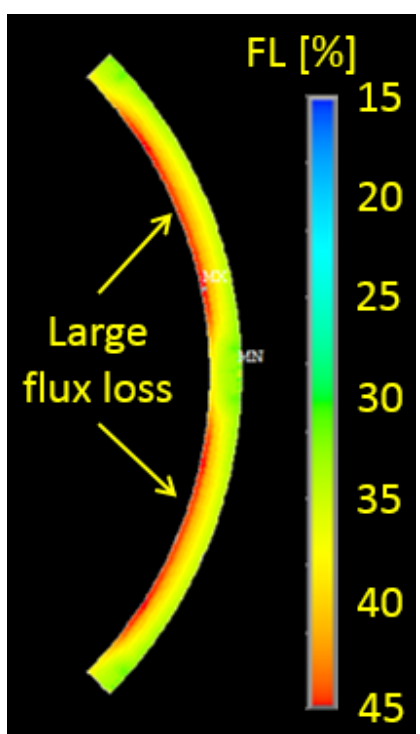

(a) Flux loss

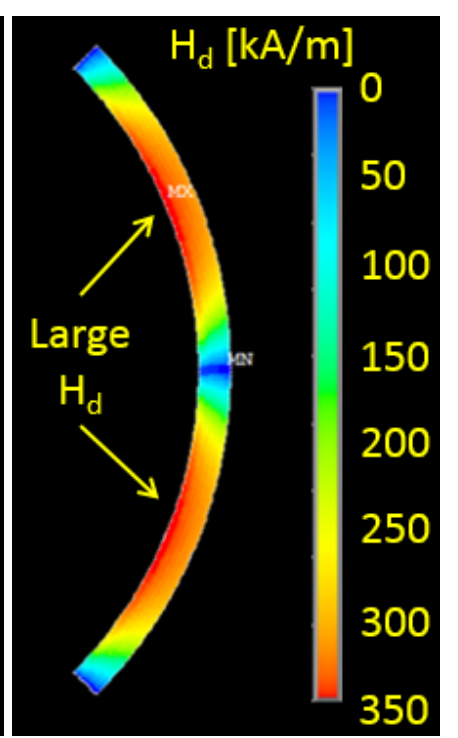

(b) Demagnetization field
Fig.5 (a) Flux loss and (b) demagnetization filed in the ring magnet made from Magnet \#1.

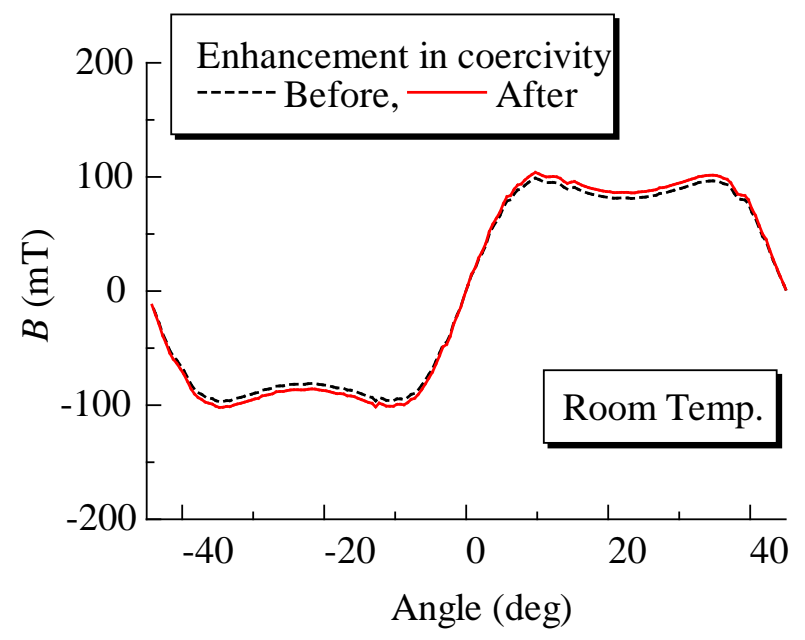

Fig.6 Flux densities on the rotor surface for the simulation models without or with locally enhanced coercivity in the ring magnet. 

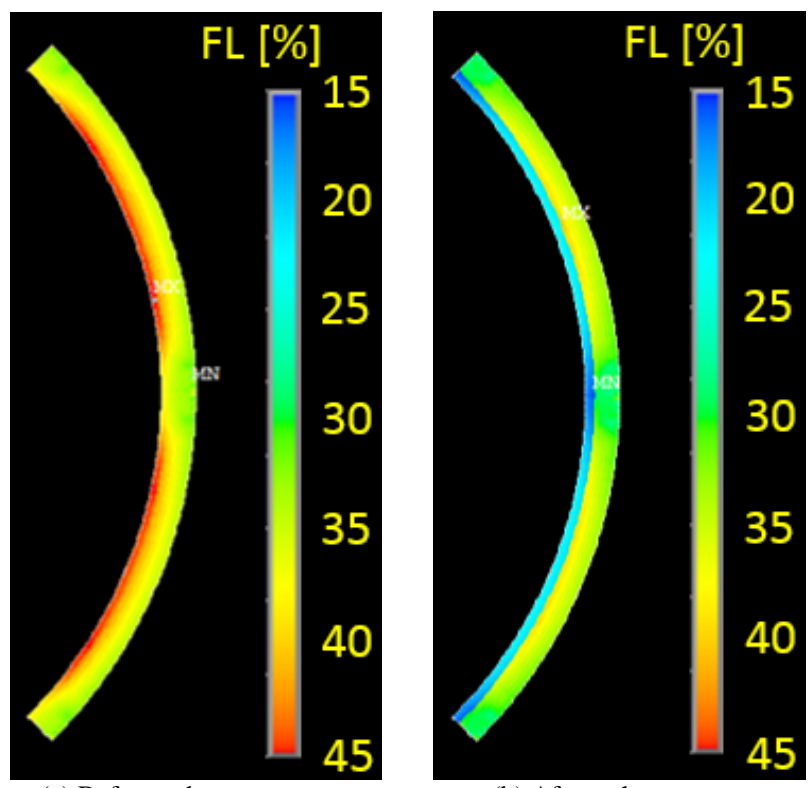

(a) Before enhancement

(b) After enhancement

Fig.7 Flux loss in the ring magnet (a) before and (b) after the enhancement in the coercivity.

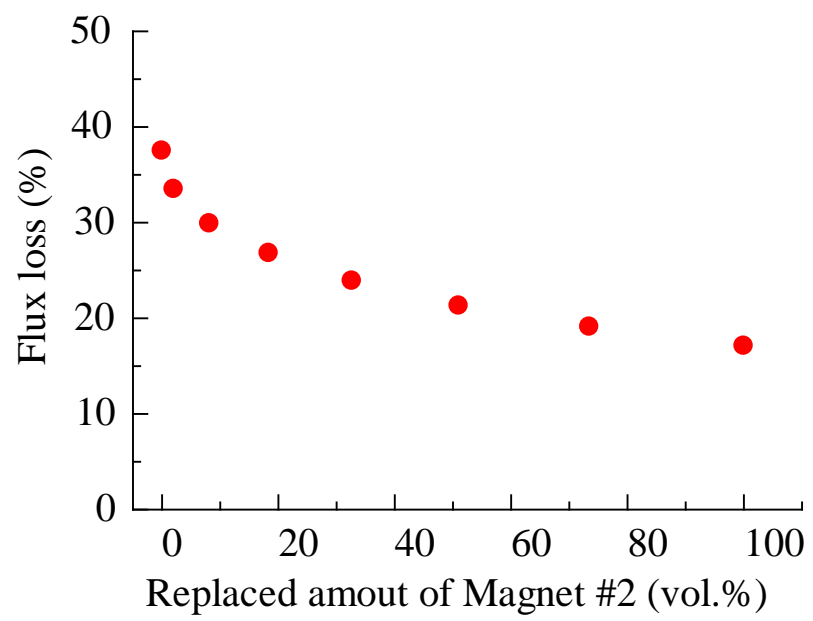

Fig.8 Flux loss of the ring magnet as a function of volume fraction of Magnet \#2.

Figure 8 shows the flux loss of the ring magnet as a function of replaced amount of Magnet \#2. The flux loss rapidly decreased by small replacement of Magnet \#2, and we found that that large replacement isn't required for the reduction in the flux loss.

From above-mentioned results, we found that locally enhanced coercivity in a Nd-Fe-B bonded ring magnet for a SPM motor is one of hopeful methods to reduce in the flux loss.

\section{CONCLUSION}

We applied our previously-proposed prediction method for the flux loss to a Nd-Fe-B bonded ring magnet for a SPM motor, and investigated the reduction in the flux loss of the ring magnet. The obtained results are summarized as follows;

(1) Large flux loss was observed in the inner side of the ring magnet.

(2) Demagnetizing filed was also, large in the inner side of the ring magnet, and this area a feels the flux loss.

(3) Local enhancement in the coercivity of the ring magnet is effective to reduce in the flux loss strongly.

(4) Large replacement of the bonded magnet with high coercivity isn't required for reduction in the flux loss.

\section{REFERENCES}

[1] M. Sagawa, S. Hirosawa, H. Yamamoto, S. Fujimura, and Y. Matsuura, "Nd-Fe-B Permanent Magnet Materials," Jpn. J. Appl. Phys., vol. 26, pp. 785-800, 1987.

[2] H. Feng, H. Chen, Z. Guo, W. Pan, M. Zhu, and W. Li, "Investigation on microstructure and magnetic properties of $\mathrm{Sm}_{2} \mathrm{Co}_{17}$ magnets aged at high temperature,” J. Appl. Phys., vol. 109, 07A763, 2011.

[3] Y. Kanai, S. Hayashida, H. Fukunaga, and F.Yamashita, "Flux Loss in Nanocomposite Magnets,” IEEE Trans. Magn., vol. 35, pp.3292-3294, 1999.

[4] H. Fukunaga, A. Toyota, N. Mine, and R. Yamamoto, "Prediction method of inhomogeneous thermal flux loss in a magnet," J. Appl. Phys., vol. 99, 08B525, 2006.

[5] H. Fukunaga, H. Koreeda, T. Yanai, M. Nakano, and F. Yamashita, "Prediction of flux loss in a Nd-Fe-B ring magnet considering magnetizing process,” J. Phys.: Conf. Ser., vol. 200, 082006, 2010.

[6] H. Fukunaga, H. Murata, T. Yanai, M. Nakano, and F. Yamashita, "Prediction method of flux loss in anisotropic NdFeB/SmFeN hybrid magnets,” J. Appl. Phys., vol. 107, 09A736, 2010.

[7] H. Fukunaga, S. Narikiyo, H. Koreeda, T. Yanai, M. Nakano, and F. Yamashita, "A method for predicting flux loss of multipole magnet and its evaluation,” IEEE Trans. Magn., vol. 47, pp.4108-4111, 2011.

[8] G. Bai, R.W. Gao, Y. Sun, G.B. Han, and B. Wang, "Study of highcoercivity sintered NdFeB magnets,” J. Magn. Magn. Mater., vol. 308 pp. 20-23, 2007.

[9] D.H. Kim, A.S. Kim, T.H. Lim, and T.S. Jang, "High coercive Nd-Fe-B sintered magnets for high temperature application,” J. Magnetics, vol. 14, pp. 27-30, 2009 